\title{
Lettre à la rédaction : Racine de Kava dans les cheveux : dosage de la kavaïne par CG-SM/SM
}

\author{
Letter to the editor : \\ Testing for kavaïne in human hair \\ by $G C-M S / M S$
}

Marion VILLAIN*, Vincent CIRIMELE, Antoine TRACQUI, Bertrand LUDES, Pascal KINTZ

Institut de Médecine Légale, 11, rue Humann - 67000 STRASBOURG - FRANCE

* Auteur à qui adresser la correspondance : Marion VILLAIN, Institut de Médecine Légale, 11, rue Humann 67000 STRASBOURG - FRANCE

Tél : 0390243356 - Fax : 0390243362 - E-mail : marion.villain@netcourrier.com

\section{Introduction}

Le kava (Piper methysticum), plante arbustiforme de quelques mètres de hauteur de la famille des Poivriers, est largement utilisé en médecine traditionnelle océanienne. En effet, l'extrait aqueux des racines de cette plante donne une boisson du même nom (kava), de couleur café au lait, responsable d'effets psychotropes et neurotropes (anxiolytique, myorelaxant et analgésique). Ces propriétés sont dues à un groupe de dihydropyrones substituées, les kava lactones, dont les principaux constituants sont la yangonine, la methysticine et la kavaïne (1).

Dans certaines îles du Pacifique, le kava est consommé quotidiennement de façon culturelle et de manière rituelle lors des cérémonies. Il est bu dans le "nakamal", au crépuscule car la lumière "tue l'esprit du kava". La dose habituelle est le contenu d'une demie noix de coco.

Depuis les années 1990, ses propriétés lui ont assuré un succès grandissant en occident. Différents extraits de kava, préconisés et prescrits essentiellement contre l'anxiété et les troubles du sommeil, devinrent disponibles en pharmacie et parapharmacie.

En 2001, le kava a été mis en cause dans plusieurs cas d'hépatites aiguës et la décision du 13 mars 2003 a porté "interdiction de la mise sur le marché à titre gratuit ou onéreux, de la délivrance et de l'utilisation à des fins thérapeutiques du kava et des produits en contenant, sous toutes formes..." (2). 
Afin de suivre sur le plan toxicologique une exposition à long terme, nous avons mis au point une méthode de dosage de la kavaïne dans les cheveux par chromatographie gazeuse couplée à la spectrométrie de masse tandem.

\section{Matériel et méthode}

Les échantillons de cheveux et de poils pubiens ont été prélevés chez un consommateur occasionnel de kava (fréquence de consommation inconnue). La mèche de cheveux provient de la zone du vertex postérieur et a été conservée dans une enveloppe, à température ambiante. L'analyse a été réalisée sur $29 \mathrm{mg}$ de cheveux, correspondant aux 2 premiers centimètres côté racine, et $50 \mathrm{mg}$ de poils pubiens. Des échantillons négatifs $(n=4)$, obtenus chez le personnel de laboratoire, ont été utilisés comme cheveux témoins.

Le dichloromethane et le méthanol sont de qualité HPLC (Merck, Darmstadt, Allemagne). La kavaïne et la methaqualone- $\mathrm{d}_{7}$ ont été respectivement fournies par Extrasynthèse (Genay, France) et Promochem (Molsheim, France).

Après décontamination des cheveux dans 2 bains successifs de dichlorométhane, l'échantillon a été coupé en fragments courts $(<1 \mathrm{~mm})$, puis incubé une heure au bain ultrasons, dans $1 \mathrm{ml}$ de méthanol en présence de 20 ng de méthaqualone- $d_{7}$ utilisée comme étalon interne (EI). Après évaporation, le résidu a été repris dans $30 \mu \mathrm{l}$ de méthanol. Ce type de préparation apparaît comme simple et universel mais nécessite l'emploi d'un détecteur hautement spécifique, ici, un spectromètre de masse tandem, du fait de chromatogrammes particulièrement chargés.

Un aliquot de $2 \mu l$ a été injecté en mode splitless (injecteur : $215^{\circ} \mathrm{C}$ ) dans une colonne Optima5-MS traversée par de l'hélium N55 à une pression de 10 psi. Le four a été programmé à une température initiale de $100^{\circ} \mathrm{C}$ pendant $1 \mathrm{~min}$, puis chauffé à $30^{\circ} \mathrm{C} / \mathrm{min}$ pour atteindre $295^{\circ} \mathrm{C}$, température maintenue pendant $6 \mathrm{~min}$. Le détecteur de masse (TSQ 700) a été utilisé en mode impact électronique avec une énergie d'ionisation de $70 \mathrm{eV}$ et une température de source de $150^{\circ} \mathrm{C}$. Les ions moléculaires $(\mathrm{m} / \mathrm{z} 230$ pour la kavaïne et $\mathrm{m} / \mathrm{z} 257$ pour le EI) ont été sélectionnés dans Q1 et les ions fils $(\mathrm{m} / \mathrm{z} 111$ et 202 pour la kavaïne et $\mathrm{m} / \mathrm{z} 242$ pour le EI) ont été détectés dans Q3 après collision avec l'argon (chambre de collision : 0,6 mTorr, énergie de collision : $-6 \mathrm{eV})$.

La courbe de calibration a été obtenue en ajoutant 5 (100 pg/mg), 10 (200 pg/mg), 20 (400 pg/mg), 50 $(1000 \mathrm{pg} / \mathrm{mg})$ et $100(2000 \mathrm{pg} / \mathrm{mg}) \mathrm{ng}$ de kavaïne à 50 $\mathrm{mg}$ de cheveux témoins (négatifs pour la kavaïne). Le coefficient de variation a été déterminé par les dosages consécutifs de 8 échantillons de $50 \mathrm{mg}$ de cheveux témoins surchargés avec de la kavaïne pour une concentration finale de $400 \mathrm{pg} / \mathrm{mg}$. La limite de détection a été évaluée à l'aide d'extraits de cheveux surchargés en quantités décroissantes de kavaïne jusqu'à un rapport signal sur bruit de fond de 3 .

\section{Résultats et discussion}

Dans ces conditions chromatographiques, il n'y avait pas d'interférence entre les analytes et des substances endogènes présentes dans les cheveux. Les ions sélectionnés ainsi que les temps de rétention de la kavaïne et de l'EI sont reportés dans le tableau I. L'ion parent de la kavaïne $(\mathrm{m} / \mathrm{z} 230)$ correspond à l'ion moléculaire ; les deux ions fils (m/z 111 et 202) ont été choisis sur des critères de spécificité et d'abondance.

La linéarité de la méthode a été vérifiée de 100 à 2000 $\mathrm{pg} / \mathrm{mg}\left(\mathrm{r}^{2}=0,998\right)$. Le coefficient de variation, à une concentration finale en kavaïne de $400 \mathrm{pg} / \mathrm{mg}$, a été mesuré à $13,7 \%$. La limite de détection a été évaluée à $30 \mathrm{pg} / \mathrm{mg}$.

L'analyse de l'échantillon d'un consommateur occasionnel de kava a permis d'identifier la kavaïne à une concentration de $418 \mathrm{pg} / \mathrm{mg}$ dans les cheveux. Une concentration supérieure $(1708 \mathrm{pg} / \mathrm{mg})$ a été détectée dans les poils pubiens (figure 1 ).

La préparation de l'échantillon est très simple. L'extraction méthanolique suivi directement par la détection par CG-SM est connue depuis le début des années 90, mais n'a été publiée qu'en 1996 par Kauert et Röhrich (3). Ces auteurs avaient alors utilisé la méthaqualone comme étalon interne, ce qui a été retenu dans notre procédure, en raison de l'absence de kavaïne deuterée sur le marché. D'après notre expérience, l'incubation méthanolique est particulièrement utile quand les liaisons cheveu-xénobiotique sont faibles. C'est le cas pour la kavaïne qui n'a pas d'atome d'azote dans sa structure, et ne peut donc pas se lier avec la mélanine chargée négativement.

La distribution des concentrations entre les cheveux et les poils pubiens est similaire à celle reportée précédemment pour d'autres composés (4), c'est-à-dire des concentrations supérieures dans les poils pubiens par rapport à celles dans les cheveux correspondants.

Tableaux I : Ions sélectionnés $(\mathrm{m} / \mathrm{z})$ et temps de rétention pour la kavaïne et l'étalon interne.

\begin{tabular}{|l|c|c|}
\hline Composés & Temps de rétention $(\mathbf{m i n})$ & Ions $(\mathbf{m} / \mathbf{z})$ \\
\hline Kavaïne & 8,60 & $230>111$ et $230>202$ \\
\hline Methaqualone- $\mathrm{d}_{7}$ (E.I.) & 8,36 & $257>242$ \\
\hline
\end{tabular}




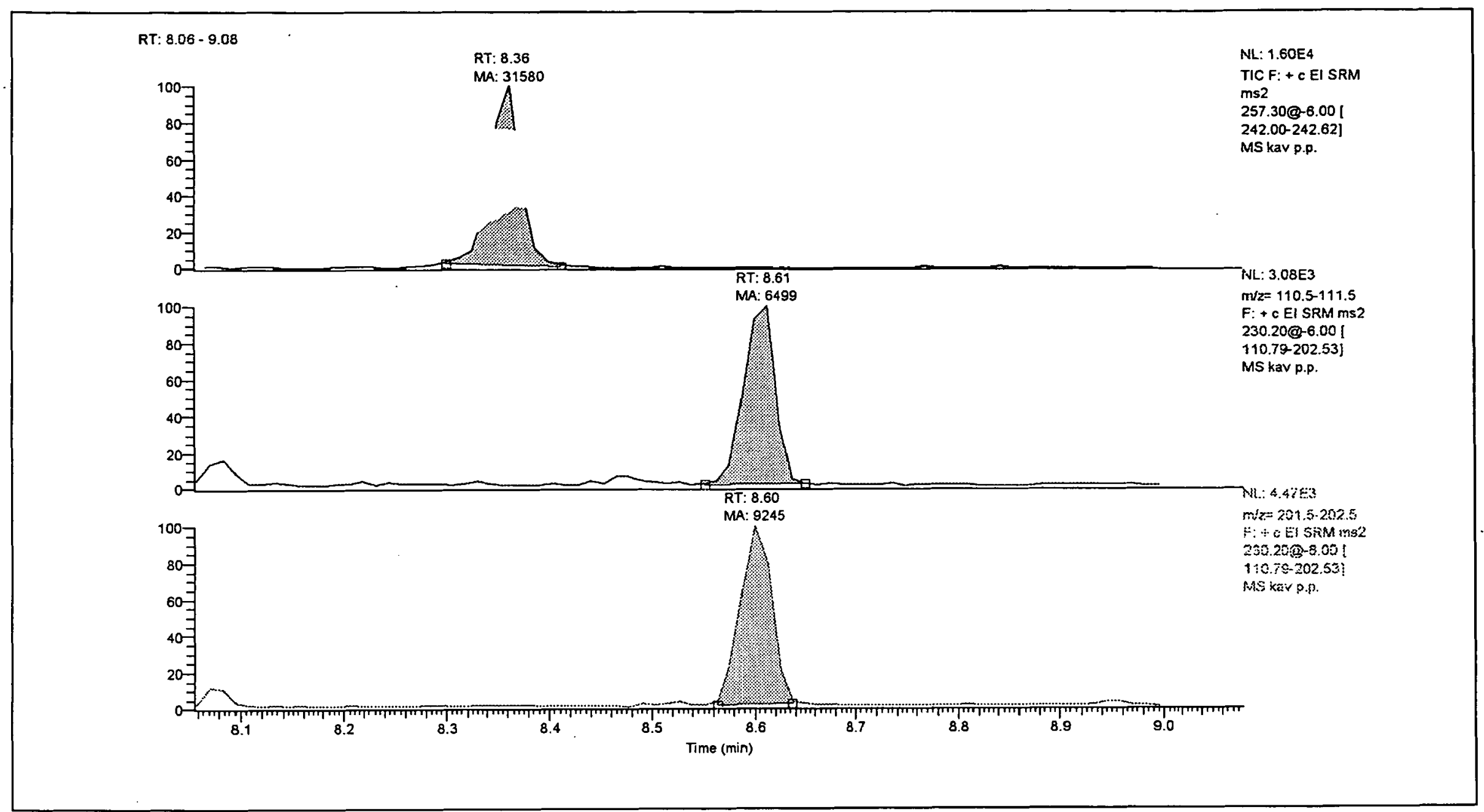

Figure 1 : Chromatogramme obtenu en mode SRM après incubation méthanolique de $50 \mathrm{mg}$ de poils pubiens d'un consommateur occasionnel de kava. La kavaïne a été quantifiée à la concentration de $1708 \mathrm{pg} / \mathrm{mg}$. En haut : ion fils de la methaqualone$d_{7}(\mathrm{~m} / \mathrm{z} 242)$, au milieu : ion fils de la kavaine $(\mathrm{m} / \mathrm{z} 111)$ et en bas : $2^{\text {ime }}$ ion fils de la kavaïne (m/z) 202).

\section{Conclusion}

La chromatographie gazeuse couplée à la spectrométrie de masse tandem, outil analytique sensible et d'une grande spécificité, permet le dosage de kavaïne dans les cheveux après une extraction peu sélective et rapide. Cette analyse complète la liste des molécules déjà identifiées dans les cheveux. Elle constitue une approche originale pour le suivi des patients présentant des troubles hépatiques en rapport avec une consommation de kava. Enfin, la polytoxicomanie associant kava, cannabis et alcool, particulièrement développée en Nouvelle-Calédonie, pourrait être documentée afin d'évaluer le profil et l'importance de l'exposition.

\section{Références}

1. Warter S. Etude de populations exposées au Kava en Nouvelle-Calédonie et à Futuna. Contribution à la connaissance de la toxicité du Kava, Thèse Doct. Médecine, Université Louis Pasteur, Strasbourg, France, 2003.

2. Décision du 13 mars 2003 portant interdiction de la mise sur le marché, à titre gratuit ou onéreux, de la délivrance et de l'utilisation à des fins thérapeutiques du kava (kavakava, kawa-kawa, Piper methysticum) et de produits en contenant, sous toutes formes, à l'exception des médicaments homéopathiques à des dilutions égales ou supérieures à la 5e dilution centésimale hahnemannienne. J.O. $\mathrm{n}^{\circ} 72$ du 26 mars 2003, p. 5367.

3. Kauert G., Röhrich J. Concentrations of delta-9-tetrahydrocannabinol, cocaine and 6-acetylmorphine in hair of drug abusers. Int. J. Legal Med. 1996 ; 108 : 294-99.

4. Mangin P., Kintz P. Variability of opiate concentrations in human hair according to their anatomical origin : hair, axillary and pubic regions. Forensic Sci. Int. 1993 ; 63 : 77-83. 